\title{
Un cadre interprétatif pour enrichir la réflexivité : le cas d'une formation à la médiation civile et commerciale
}

An interpretative framework for enhancing reflexivity: the case of civil and commercial mediation training

Valérie Saint-Dizier de Almeida, Flora Ilardo, Isabelle Serre, Olivier Cachard et Guy Deloffre

\section{OpenEdition \\ Journals}

Édition électronique

URL : http://journals.openedition.org/activites/4678

DOI : $10.4000 /$ activites.4678

ISSN : $1765-2723$

Éditeur

ARPACT - Association Recherches et Pratiques sur les ACTivités

\section{Référence électronique}

Valérie Saint-Dizier de Almeida, Flora Ilardo, Isabelle Serre, Olivier Cachard et Guy Deloffre, « Un cadre interprétatif pour enrichir la réflexivité : le cas d'une formation à la médiation civile et commerciale ", Activités [En ligne], 16-2 | 2019, mis en ligne le 15 octobre 2019, consulté le 17 octobre 2019. URL http://journals.openedition.org/activites/4678; DOI : 10.4000/activites.4678

Ce document a été généré automatiquement le 17 octobre 2019

\section{cc)}

Activités est mis à disposition selon les termes de la licence Creative Commons Attribution - Pas d'Utilisation Commerciale - Pas de Modification 4.0 International 


\title{
Un cadre interprétatif pour enrichir la réflexivité : le cas d'une formation à la médiation civile et commerciale
}

\author{
An interpretative framework for enhancing reflexivity: the case of civil and \\ commercial mediation training
}

Valérie Saint-Dizier de Almeida, Flora Ilardo, Isabelle Serre, Olivier Cachard et Guy Deloffre

\section{NOTE DE L'ÉDITEUR}

Article accepté le 30 mai 2018, accepté le 12 février 2019

\section{Introduction}

Pour la formation à des activités professionnelles requérant le développement de compétences communicationnelles, on note l'emploi de dispositifs de formation visant également à promouvoir la réflexivité des apprenants. C'est le cas par exemple de formations en École Supérieure du Professorat et de l'Éducation (ESPE) vouées à former à la gestion de la classe, à la régulation des conflits... (Laurent, \& Saujat, 2015 ; Perrenoud, 1999, 2004 ; Vacher, 2011) et de formations à la conduite des entretiens d'annonce de diagnostics dans le champ médical (Butow, Cockbum, Girgis, Bowman, Schofield, D’Este et al., 2008 ; Cuenot, Cochand, Lanares, Feihl, Bonvin, Guex et al., 2005). Pour faire acquérir les compétences communicationnelles requises et/ou des compétences à la réflexivité, les formateurs exploitent la technique du jeu de rôle ou des vidéos de l'activité autour desquelles les apprenants sont invités à échanger. Le rôle du formateur consiste généralement à affecter les prises de parole et à animer, guider 
les échanges autour de l'activité en se basant sur des connaissances académiques qui constituent un cadre pour l'interprétation des pratiques.

Dans le cadre de cet article, l'enjeu est de présenter et de tester un nouveau cadre pour l'interprétation de pratiques professionnelles qui s'expriment à travers des interactions langagières. Le cadre emprunte aux théories, modèles, concepts et méthodologies issus des sciences du langage et de la psychologie de la communication. Il est mobilisé ici dans le cadre d'une formation à la médiation civile et commerciale. La médiation est une activité professionnelle qui se matérialise à travers des communications interpersonnelles (en l'occurrence, des trilogues - communications à trois) et qui s'inscrit dans le champ des relations de service (Cerf, \& Falzon, 2005). Une partie de la formation vise à conduire les apprenants à mener une réflexion collective sur l'activité de médiation. Elle est prise en charge par une formatrice à la médiation qui utilise dans le cadre de sa pratique de formation, la méthode du jeu de rôle suivie de discussions collectives. Pour guider les discussions collectives, elle exploite des connaissances académiques sur la médiation : le scénario de la médiation, des prescriptions comme : le médiateur doit laisser chaque partie s'exprimer, il doit être impartial, il doit reformuler les propos tenus par chacune des parties, etc. Le dispositif qu'elle utilise habituellement est similaire à ceux visant le développement de compétences à la réflexivité. Effectivement, en référence aux travaux de Bouissou et Brau-Antony (2005), son dispositif mobilise :

1. un support propice à discussion (l'activité accomplie en jeu de rôle);

2. des savoirs formels (des connaissances académiques sur la médiation)

3. une discussion collective sur l'activité produite.

Dans le cadre de cette étude exploratoire, il s'agit donc, de mobiliser en complément ce nouveau cadre (le présenter aux apprenants et montrer son applicabilité à travers l'analyse de productions langagières émises dans un jeu de rôle simulant une médiation) et d'apprécier si ce cadre et les résultats d'analyse auxquels il aboutit, permettent d'enrichir les discussions collectives.

3 La partie théorique rend compte de travaux et de formations qui visent le développement de compétences communicationnelles et/ou à la réflexivité. La deuxième partie est consacrée à l'étude de cas ; elle présente dans un premier temps le dispositif et les ressources mobilisées (le cadre interprétatif et les résultats de l'analyse) et dans un second temps, elle met en exergue l'impact de la communication de ces résultats sur les productions émises en discussion collective. L'article se poursuit par une discussion et une conclusion montrant l'intérêt de ce nouveau cadre interprétatif et les limites de cette étude.

\section{Cadre théorique}

\subsection{La formation aux activités qui prennent forme dans les interactions langagières}

Former aux activités professionnelles qui s'expriment à travers des interactions langagières est complexe pour plusieurs raisons. La dynamique des communications interpersonnelles est difficile à cerner : c'est par et au moyen du langage qui possède sa propre logique (Falzon, 1994) que s'opèrent de manière simultanée la gestion socio- 
émotionnelle et l'accomplissement de la tâche comportant également leurs propres contraintes. Aussi, ces activités prennent forme en situation dynamique (Hoc, 1996) ; de fait, il est impossible d'anticiper ce que vont produire les protagonistes au gré de l'interaction. Le psychologue ergonome doit alors se résoudre à déterminer et à former aux composants et déterminants impliqués dans l'activité (Lancry-Hoesland, \& Laville, 2004); ce qui demeure insuffisant pour faire acquérir les compétences communicationnelles sous-jacentes (Saint-Dizier de Almeida, 2013).

Une option relativement récente déployée notamment dans le champ de la formation des professeurs des écoles - formations à l'animation/gestion de classe - (Vacher, 2011, op. cit. ; Laurent, \& Saujat, 2015, op. cit.) et dans celui de la formation des médecins formation à la conduite d'entretien d'annonce de diagnostics - (Butow et al., 2008, op. cit.; Cuenot et al., 2005), repose sur l'emploi de la technique du jeu de rôle suivi de discussions collectives. Les discussions sont animées et guidées par un formateur qui exploite pour ce guidage des connaissances académiques. Par exemple pour la formation à la conduite d'entretien d'annonce de diagnostics médicaux, il peut se fonder sur des prescriptions et proscriptions utilisées pour faire acquérir la démarche empathique (écoute active et attitude compréhensive ; cf. 1). On forme à l'identification de signes révélant l'expression d'émotions. Plus précisément, on apprend aux formés à être attentif au regard des patients, à un changement de teint, à leurs mouvements, à la tonalité de la voix, aux peurs qu'ils expriment et craintes qu'ils communiquent. Ces formations visent également à rectifier le comportement des formés, à les conduire à développer les techniques de relance et les comportements qui sont au cœur de l'écoute active et de l'attitude compréhensive (Rogers, 1942, 2008). Ces formations exploitent, au cours du processus pédagogique, également un format davantage déclaratif où les prescriptions et proscriptions à la base des rectifications de comportements sont précisées: comprendre le patient, ne pas le juger, ne pas le sermonner, être attentif à ses émotions, lui faire verbaliser ses émotions et ses états mentaux, montrer qu'on l'écoute et qu'on le comprend.

Tableau 1 : Prescriptions et proscriptions reliées à « adopter une démarche empathique ", extrait de Saint-Dizier de Almeida (2013).

Table 1: Prescriptions and proscriptions related to "adopt an empathetic approach" extract from SaintDizier de Almeida (2013)

\begin{tabular}{|c|c|c|c|c|}
\hline \multicolumn{5}{|c|}{ Adopter une démarche empathique } \\
\hline $\begin{array}{c}\text { Favoriser } \\
\text { 1'expression du } \\
\text { patient }\end{array}$ & Etre attentif & $\begin{array}{c}\text { Montrer qu'on } \\
\text { l'écoute }\end{array}$ & $\begin{array}{c}\text { Comprendre le } \\
\text { patient }\end{array}$ & $\begin{array}{l}\text { Montrer qu'on le } \\
\text { comprend }\end{array}$ \\
\hline $\begin{array}{l}\Rightarrow \mathrm{Ne} \text { pas } \\
\text { l'interrompre } \\
\Rightarrow \text { Accepter des } \\
\text { silences } \\
\Rightarrow \text { Susciter } \\
\text { l'expression par des } \\
\text { relances }\end{array}$ & $\begin{array}{l}\Rightarrow \text { Ecouter le patient } \\
\Rightarrow \text { Etre attentif au } \\
\text { non verbal } \\
\Rightarrow \text { Identifier les } \\
\text { émotions }\end{array}$ & $\begin{array}{l}\Rightarrow \text { Choisir une } \\
\text { proximité adéquate } \\
\Rightarrow \text { Utiliser des } \\
\text { signes verbaux } \\
\text { (phatiques) et non } \\
\text { verbaux (regard...) }\end{array}$ & $\begin{array}{l}\Rightarrow \text { Le questionner } \\
\text { par des relances en } \\
\text { écho, en reflet, des } \\
\text { reformulations, des } \\
\text { questions }\end{array}$ & $\begin{array}{l}\Rightarrow \text { Le soutenir } \\
\Rightarrow \mathrm{Ne} \text { pas l'évaluer } \\
\Rightarrow \mathrm{Ne} \text { pas le juger } \\
\Rightarrow \mathrm{Ne} \text { pas le } \\
\text { sermonner }\end{array}$ \\
\hline
\end{tabular}

6 Les formations destinées aux professeurs des écoles peuvent reposer sur des cadres exploitant des référentiels de compétences (Perrenoud, 1999, op. cit.); par exemple, les dix domaines de compétences reconnues comme prioritaires dans la formation des enseignants - cf. annexe de l'article Perrenoud, 1999, op. cit.). 
7 Ce type de dispositif (discussion collective consécutive à des jeux de rôle) est utilisé en formation initiale afin de parfaire la pratique en acquisition (Cuenot, et al., 2005, op. cit. ; Butow et al., 2008, op. cit. ; Vacher, 2011, op. cit.) et également en formation continue pour optimiser la pratique installée (Laurent, \& Saujat, 2015, op. cit.).

8 Ces situations de formation sont particulièrement propices au développement de compétences à la réflexivité; elles montrent notamment comment interroger une pratique professionnelle.

\subsection{La réflexivité}

9 Si la réflexion est un processus cognitif mobilisé pour le traitement d'une situation qui se limite à l'analyse de cette dernière, la réflexivité constitue néanmoins un "saut épistémologique » en englobant la réflexion sur la situation et la réflexion sur la réflexion (Donnay, \& Charlier, 2006).

10 La réflexivité renvoie à «la capacité d'un praticien de prendre sa propre pratique comme objet de réflexion, voire de théorisation" (Perrenoud, 2001, p. 9). Les professionnels réflexifs sont aptes «à rendre compte de leurs pratiques et les expliciter» (Bouissou, \& Brau-Antony, 2005, p. 114); ils deviennent ainsi acteurs de leur pratique (Perrenoud, 1999, op.cit.). La réflexivité repose sur le développement de compétences qui s'acquièrent progressivement au gré de la pratique réflexive : «c'est en pratiquant une activité de réflexivité que l'on exprime et construit des compétences de réflexivité » (Bouissou, \& Brau-Antony, 2005, op. cit., p. 116). Le développement de compétences à la réflexivité permet de passer du «faire » à l'« agir » (Boutinet, 1998) à la différence de l'agir, le faire renvoie à une pratique qui n'a pas fait l'objet d'une activité réflexive recourant à des cadres interprétatifs académiques (Perrenoud, 1999, op. cit.).

11 La réflexivité est une notion devenue incontournable en sciences de l'éducation, elle constitue un enjeu majeur des ingénieries de formation (Guillaumin, 2009). Dans ce champ disciplinaire, cette notion a été introduite dans les années 80 par Schön (1987) à travers le vocable de " praticien réflexif » et a donné lieu à un courant de recherche qui vise par exemple à formaliser les différentes étapes du développement de la capacité à la réflexivité ou encore à construire des grilles permettant d'évaluer son développement (Roy, 2008). En psychologie ergonomique, cette notion est également mobilisée notamment dans une perspective développementale: conduire les opérateurs à optimiser leur pratique (Mollo, \& Falzon, 2004). Ceci suppose des prises de conscience et des échanges avec d'autres professionnels permettant d'envisager d'autres manières de faire. Les prises de conscience requièrent que l'opérateur ait accès à son activité telle qu'elle s'accomplit en situation. Des méthodologies sont développées pour permettre cet accès : l'entretien d'explicitation développé par Vermersch (1994, 2014) conduit le professionnel à (re)vivre au niveau sensoriel la situation qu'il évoque et à rester dans la description de son activité ; la méthode de l'auto-confrontation permet au professionnel en situation d'entretien de visualiser le film de son activité. La vidéo n'est pas le seul support propice à la réflexivité, les traces de son activité (chroniques d'activité, d'historiques constituées à partir d'indicateurs divers, cartes dynamiques...) peuvent également servir de support aux verbalisations (Cahour, \& Licoppe, 2010). Ces méthodologies permettent des prises de conscience, d'accéder à des composants pré-réfléchis (Theureau, 1992) - ce sont des composants pas 
immédiatement accessibles à la conscience réfléchie, mais qui peuvent l'être en situation d'évocation ou en situation d'auto-confrontation (Cahour, 2006) - et suscitent de la réflexivité (Clot, Faïta, Fernandez, \& Scheller, 2001 ; Mollo, \& Falzon, 2004 ; SixTouchard, 1998 ; Theureau, 2004). La méthode de l'auto-confrontation croisée (Clot et al. 2001, op. cit.) qui consiste à faire verbaliser en entretien l'opérateur filmé et un autre opérateur face à la vidéo de l'activité du premier et de l'allo-confrontation où il s'agit de faire échanger un collectif d'opérateurs autour d'une activité filmée (Mollo, \& Falzon, 2004, op. cit.) permettent de confronter des pratiques et à chacun d'envisager des optimisations de sa propre pratique.

Les composants de l'activité mis en discussion en auto ou allo-confrontation peuvent relever tant de la sphère opératoire/instrumentale que de la sphère socioémotionnelle - en référence à la taxonomie de Bales (1972). Ainsi les échanges, les prises de conscience et les réflexions peuvent avoir pour objet des connaissances ou des affects (Cahour, 2006, op. cit. ; Prost, Cahour, \& Détienne, 2014 ; Saint-Dizier de Almeida, 2015). Dans les formations à l'annonce de diagnostics, le focus est davantage mis sur l'expression et la régulation des émotions. Dans le champ de l'éducation, on peut mentionner un dispositif conçu pour former à la régulation d'interactions éprouvantes émotionnellement et potentiellement conflictuelles (Vinatier, \& Marfizi, 2018) - une partie du dispositif vise à conduire les apprenants à prendre conscience de la dimension émotionnelle de productions langagières et à exprimer leur vécu subjectif face à l'émotion exprimée.

13 En référence à Bouissou et Brau-Anthony (2005, op.cit.), les dispositifs visant le développement de compétences à la réflexivité nécessitent :

1. un support approprié, une activité qui puisse être observée. L'activité constitue l'objet sur lequel la réflexivité va pouvoir s'exercer. L'activité peut avoir été produite via la mise en place de jeu de rôle ou filmée en situation authentique de travail. La visualisation du film permet une distanciation avec l'action (Mollo, \& Nascimento, 2013). Cette " mise à distance » permet une analyse critique de l'action ou de la situation (Falzon, 1994, op. cit.). C'est « la situation de confrontation qui fournit une occasion instrumentée de faire retour sur son expérience, de s'y arrêter quelque temps, de s'extraire du feu de l'action dans laquelle le vécu était inscrit pour déplier ce qui s'y passe, en le mettant en mots, en prenant le temps d'essayer de retrouver finement ce qui s'y déroulait, d'analyser, comparer, évaluer pour produire et reformuler le sens de l'activité [...] » (Cahour, \& Licoppe, 2010, op. cit., p. 247).

2. la transmission de savoirs formalisés, qui constituent des outils conceptuels utiles pour désigner leur pratique, la comprendre, y réfléchir. Il s'agit de fournir un langage que les formés vont pouvoir mobiliser " pour désigner leur pratique (pratique d'abord vécue et non formalisée), la comprendre, au-delà de leurs seules convictions ou expériences passées » (Cahour, \& Licoppe, 2010, op.cit., p. 116). Le langage met à disposition des cadres interprétatifs qui permettent de faire prendre conscience aux formés que le réel peut être approché de différentes manières - «si (...) nous analysons d'une certaine manière (un événement), cela n'interdit pas d'autres manières de l'expliquer» (Bruner, 1996, p. 115). Comme mentionnés dans la partie précédente, dans le champ de la formation des enseignants, les référentiels de compétences peuvent constituer une base au développement de cadres interprétatifs ; dans le champ de formation à l'annonce de diagnostics médicaux, ce sont notamment les travaux de Rogers $(1942,2008$, op. cit.) sur la démarche empathique qui ont permis de développer un cadre interprétatif (cf. Tableau 1).

3. le recours à des discussions collectives. La dimension collective de la réflexivité a un rôle important dans le développement de la capacité réflexive d'un individu. "La pratique réflexive collective est plus que l'addition de réflexions individuelles, elle a un double effet 
individuel et collectif»(Mollo, \& Nascimento, 2013, op.cit., p.10): le simple fait de communiquer en groupe sur l'expérience de réalisation d'une action permet «la déconstruction de l'expérience et la reconstruction d'un sens partagé qui transforme les compréhensions et change la pratique » (Crow, \& Smith, 2005 cité par Correa Molina, Collin, Chaubet, \& Gervais, 2010, p. 144). Plus précisément, les discussions collectives permettent aux individus de prendre connaissance des autres points de vue (Mollo, \& Nascimento, 2013, op. cit.) et de confronter des idées et des modes de conceptions divergentes; c'est le traitement dialogique des controverses qui conduit à réévaluer et enrichir les savoirs et savoir-faire (Clot, 1999). Les discussions collectives vont ainsi conduire les acteurs à réviser leur propre point de vue sur l'activité, en le modifiant, en le complétant (Mollo, \& Nascimento, 2013, op. cit.).

\section{L'Étude de cas}

\subsection{La formation à la médiation : le dispositif et le processus de formation}

14 le certificat national à la médiation civile et commerciale. Cette formation certifiée a été conçue par Cachard et Deloffre en 2016, dans le cadre d'une coopération entre la Faculté de Droit de l'Université de Lorraine et l'Institut de Commerce de Nancy Business School.

Elle comporte 55 heures de formation: des enseignements magistraux dispensés par des juristes, économistes et des travaux pratiques animés par une formatrice à la médiation qui est également médiatrice. Dans sa pratique, elle privilégie la technique des jeux de rôle pour faire acquérir la pratique de médiation. Une psychologue ergonome spécialisée dans l'étude des communications interpersonnelles a été sollicitée pour assurer en partenariat avec la formatrice à la médiation, sept heures de formation (deux séances de $3 \mathrm{~h} 30$ ). La première séance est programmée en deuxième journée de formation. La seconde séance a eu lieu deux semaines après la première.

Quinze apprenants ont bénéficié de cette formation. Ils disposent avant la formation de connaissances sur l'enjeu d'une médiation et la fonction du médiateur. Les verbatim collectés au début de la première séance tels «ce sont les parties qui trouvent une solution, elles sont guidées pour cela par le médiateur ", « le médiateur doit être neutre et impartial » traduisent la mobilisation de connaissances conformes aux prescriptions propres à la médiation civile et commerciale (cf.par exemple Smets-Gary, \& Becker, 2016).

La première séance comporte quatre étapes.

1. Elle débute par un tour de table. La formatrice à la médiation demande aux apprenants de dire en quelques phrases la façon dont ils perçoivent l'activité de médiation.

2. Ensuite, un jeu de rôle est mis en place impliquant trois apprenants volontaires (deux jouent le rôle des parties ; le troisième, le rôle de médiateur). Le jeu de rôle est filmé. Le scénario est le suivant: deux amies (Marie et Catherine) qui avaient fait l'achat d'un commerce ne s'entendent plus ; Marie souhaite racheter les parts de Catherine, cette dernière s'y refuse. Une médiation est alors demandée par Marie. Le scénario a été élaboré par la formatrice à la médiation sur la base de cas provenant de médiations dans le domaine civil et commercial. Les trois volontaires se voient transmettre une feuille précisant, le contexte de la médiation, le rôle attendu. Avant le jeu de rôle, la formatrice s'assure que chaque acteur du jeu a bien 
compris le scénario et les consignes. Elle explique aux acteurs jouant les "parties » que le but est de permettre à l'apprenti médiateur de montrer ce qu'il sait faire et que l'idée n'est pas de bloquer systématiquement les débats en restant sur ses positions ou, au contraire, de faciliter le travail du médiateur en essayant de venir le " sauver ». Les observateurs ont pour consigne de ne pas réagir pendant la durée du jeu de rôle : ne pas rire ou montrer sa surprise ou être choqué ou autre.... La formatrice les invite toujours à rester concentrés fermement quand ça déborde... et à noter ce qui présente à leurs yeux de l'intérêt pour le partager en discussion collective.

3. À l'issue du jeu de rôle, la formatrice accueille en premier lieu le ressenti de l'apprenti médiateur puis celui des "parties » de façon à ce qu'ils « ressortent chacun doucement du jeu psychologique». Ils doivent rester à leur place tant que cette partie du débriefing n'est pas terminée. Ensuite, la formatrice invite les observateurs à s'exprimer sur la base de ce qu'ils ont relevé lors de l'observation. La formatrice utilise une posture qui est celle du médiateur : neutre, impartiale et indépendante et invite implicitement le groupe à observer sa posture et sa pratique qui mobilise les outils qu'elle préconise et présentera plus précisément au cours des séances de cours ultérieurs : l'écoute active et activante, la noninterruption, le non-jugement, ne pas répondre aux questions posées, mais guider activement celui qui a posé la question de façon à ce qu'il trouve lui-même sa propre réponse en fonction de sa vérité.

4. En fin de séance, la psychologue ergonome présente (sous forme de cours magistral) des théories et modèles de la communication interpersonnelle qui constituent un cadre interprétatif permettant d'accéder à certaines dimensions de l'activité communicationnelle. Pour justifier le cours à dispenser, la psychologue ergonome précise aux formés que la médiation est une activité qui se matérialise à travers des communications interpersonnelles, que pour pouvoir échanger à propos de cette activité qui se matérialise dans le discours, il est utile d'avoir des connaissances sur la communication interpersonnelle.

La seconde séance comporte deux périodes.

1. Elle débute par une allo-confrontation collective sur la base des vingt premières minutes de la vidéo du jeu de rôle. La discussion est animée par la formatrice à la médiation qui gère l'allocation des prises de parole, produit des relances. Les consignes et sa posture sont similaires à celles utilisées lors de la phase précédente.

2. Au cours de la deuxième période, la psychologue ergonome qui a effectué l'analyse des productions émises en jeu de rôle grâce au cadre présenté ci-après intervient en reprenant des propos tenus lors des discussions collectives afin de les enrichir des résultats d'analyse ; le collectif est invité à réagir à l'apport de ces résultats.

\subsection{Les ressources mobilisées : le cadre interprétatif et les résultats d'analyse du jeu de rôle}

\subsubsection{Le cadre pour l'étude de la dimension communicationnelle de l'activité}

Le cadre théorique mobilisé pour l'étude des communications s'inscrit dans une approche pragmatique et dialogique du discours. Pour favoriser la compréhension de cette posture épistémologique qui traduit le passage du paradigme de la communicativité, à celui de la communicabilité (Brassac, 2001), nous débutons en présentant aux apprenants les modèles de la communication qui se sont succédé dans le temps: du modèle du code de Shannon et Weaver (1949) au modèle de l'enchaînement conversationnel (Trognon, \& Brassac, 1992). Il s'agit de faire prendre conscience que le langage naturel ne fonctionne pas comme un code, que les mots ne 
sont pas univoques, que leur signification dépend du contexte (approche pragmatique), que le sens d'un énoncé se co-construit à rebours dans l'interlocution (approche dialogique) - i.e. ce qui signifie que l'intention de sens qu'un locuteur souhaite communiquer peut ne pas être identifiée et peut requérir des échanges supplémentaires visant un partage de l'intention initiale. Ces propos sont illustrés par des séquences.

Le focus est également mis sur le fait que communiquer, ne se réduit pas à énoncer des propos (le fait de dire - niveau de la locution), mais c'est également agir (niveau actionnel : affirmer, faire une requête, une promesse, etc.), et produire un effet sur l'allocutaire - celui qui écoute et interprète l'énoncé- (niveau perlocutoire/ interactionnel : le convaincre, lui faire faire quelque chose, etc.) (Austin, 1962).

21 Le focus est également mis sur les éléments du contexte - contexte pris dans son acception la plus large - à prendre en compte pour accéder au sens des énoncés. En l'occurrence le cadre institutionnel, l'enjeu de l'interaction, les statuts des partenaires, le contrat de communication (c'est un contrat par lequel les participants à une interaction acceptent un certain nombre de règles et de principes rendant possible l'interaction; il permet à chaque interactant de se faire une idée sur ses droits, ses devoirs et ceux de l'autre (Charaudeau, 1983)), le contexte physique et le cotexte (ce qui a été déjà produit en communication) et surtout la séquence dans laquelle s'insèrent les énoncés (Lacoste, 1983; Whalen, Zimmerman, \& Whalen, 1992).

Des modèles permettant d'approcher certaines dimensions de l'activité communicationnelle leur sont ensuite présentés.

23 L'approche de la dimension structuro-fonctionnelle des communications repose sur la thèse selon laquelle toute activité est structurée et cette structure transparait dans les communications (Kostulski, \& Trognon, 1998). Il est fait référence au modèle développé par l'École de Genève qui permet de formaliser la structuration fonctionnelle des communications (Roulet, Auchlin, Moeschler, Rubbattel, \& Schelling, 1985), notamment la succession des phases opératoires et leur articulation. Ce type d'analyse permet d'apprécier si la structuration de la communication est conforme ou non à l'attendu, au prescrit.

24 Concernant la dimension contractuelle, nous précisons qu'il faut distinguer le contrat de communication externe et le contrat de communication co-construit. Le premier renvoie au contrat théorique/officiel/prescrit. Il peut faire l'objet d'une explicitation en début d'interaction. Le contrat renseigne sur «ce qui peut être dit ou fait dans une situation donnée, de connaître les objets que l'on peut "mettre en communication" ainsi que la manière de le faire » (Vion, 1992, p. 74). Le contrat co-construit renvoie au contrat qui prend forme au gré des interactions langagières (Camus, 1999), il peut ne pas être en adéquation avec le contrat externe (Saint-Dizier de Almeida, 2009).

25 La dimension relationnelle (socio-émotionnelle) est amorcée en référence aux travaux de l'École de Palo Alto (Watzlawick, Beavin, \& Jackson, 1972) posant que communiquer requiert simultanément la production d'un contenu et la gestion de la relation. La relation peut s'étudier à travers :

1. l'étude des rôles qui consiste à identifier les actions opératoires et à les rapporter au statut du locuteur (Saint-Dizier de Almeida, 2009, op. cit.) ;

2. l'étude des rapports de place (relation verticale - Kerbrat-Orecchioni, 1988) qui repose sur l'identification de la valeur taxémique des énoncés (par exemple une requête d'action place le locuteur en position haute et simultanément l'allocutaire en position basse - i.e. le 
locuteur se donne le pourvoir de donner un ordre à l'allocutaire qui se trouve de fait en position d'exécutant) ;

3. l'étude de la proximité/distance entre les membres (relation horizontale - Marcoccia, 2007) qui consiste à étudier les pronoms d'allocution, les choix lexicaux, syntaxiques, grammaticaux.

Les émotions sont abordées en référence notamment aux travaux de Cahour et Lancry (2011) qui distinguent les émotions simples versus complexes à valence négative versus positive. Des exemples sont mobilisés pour traiter de la matérialité des émotions. Nous traitons également des énoncés qui ont pour fonction perlocutoire de provoquer des émotions chez l'allocutaire (Saint-Dizier de Almeida, 2013, op. cit.).

\subsubsection{Les résultats d'analyse du jeu de rôle}

Le jeu de rôle a été filmé, transcrit et analysé en référence au cadre précédent.

\section{La structure fonctionnelle de l'activité}

Figure 1 : La structure fonctionnelle de l'activité de médiation en jeu de rôle. Figure 1: The functional structure of the mediation activity in role-play

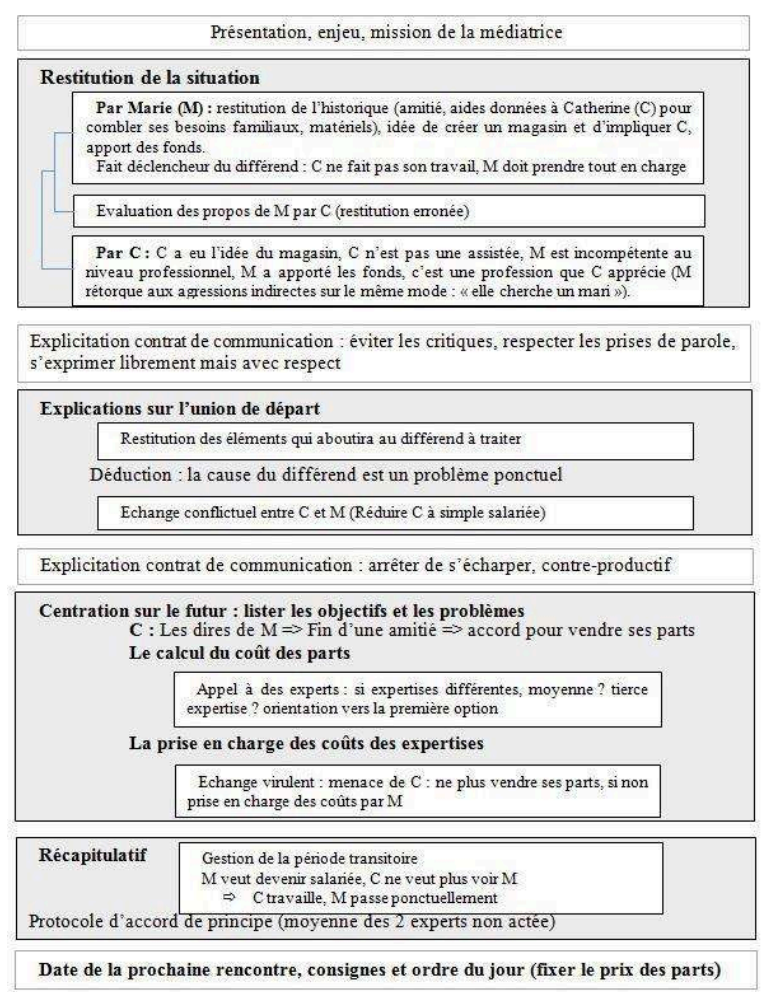

Légende : Marie (M) et Catherine (C) sont les deux parties qu'un différend oppose. Les encadrés en blanc renvoient à des séquences qui ont une fonction socio-organisationnelle ; les encadrés en grisé renvoient à des séquences qui ont une fonction instrumentale/opératoire.

Legend: Marie (M) and Catherine $(C)$ are the two parties in disagreement. The white boxes refer to sequences that have a socio-organizational function; the gray boxes refer to sequences that have an instrumental/operative function

L'analyse structuro-fonctionnelle permet de visualiser les différentes étapes qui se sont succédé au cours de la médiation. Chaque encadré donne des indications sur les contenus en discussion au cours de ces différentes étapes. Les longs encadrés en blanc 
renvoient à des séquences qui visent à expliciter, réexpliquer le contrat de communication (respecter la parole d'autrui) - sphère socio-organisationnelle (Bales, 1972, op. cit.). Ceux en grisé, renvoient à des séquences où les énoncés concourent davantage à la progression du processus de médiation - sphère instrumentale/ opératoire (Bales, 1972, op. cit.).

La première phase est prise en charge par l'apprentie médiatrice qui présente ce qu'est une médiation, son enjeu, la mission de la médiatrice (cf. $1^{\text {er }}$ encadré blanc).

La phase suivante « restitution de la situation » est amorcée par l'apprentie médiatrice et va permettre à chaque partie de restituer la situation de son point de vue. On notera que Catherine qui intervient en second pour restituer son récit, va, sans y être invitée, manifester ses désaccords quant à des propos tenus par Marie lors de son récit. On notera également qu'au cours des deux récits, chaque partie n'hésite pas à lancer des piques à l'autre, ce qui conduira l'apprentie médiatrice à initier une phase visant à expliciter le contrat de communication propre à la médiation: ne pas s'insulter, dénigrer (cf. $2^{\mathrm{e}}$ encadré blanc de la Figure 1 ).

31 La phase intitulée "explications sur l'union de départ » permet un travail sur les facteurs qui ont conduit au différend. Cette phase est encore marquée par des affrontements, des insultes qui conduiront l'apprentie médiatrice à opérer une suspension pour signaler à nouveau qu'en médiation, on ne s'insulte pas, on ne dénigre pas que l'enjeu est d'aboutir à un consensus (cf. $3^{\mathrm{e}}$ encadré blanc de la Figure 1).

Elle amorce ensuite une phase visant à trouver des solutions. Les séquences conflictuelles produites lors de la phase précédente conduisent Catherine à revenir sur sa décision initiale : elle est finalement d'accord pour vendre ses parts pour ne plus être en contact avec Marie. Se pose alors la question de l'estimation du coût des parts. Marie propose de solliciter un expert; Catherine n'est pas d'accord avec cette option et souhaite deux experts, un sollicité par Marie, l'autre par elle-même. Se pose ensuite la question d'une éventuelle différence entre les deux estimations. Au terme de cet échange, les deux parties envisagent de faire la moyenne des deux expertises sans être toutes deux convaincues par cette option.

33 S'ensuit une phase récapitulative qui permet également de traiter de la phase de transition ; Catherine souhaitant, pour des raisons financières, poursuivre son activité professionnelle, mais sans être en contact avec Marie.

34 La médiation est clôturée par l'apprentie médiatrice qui précise l'ordre du jour de la prochaine rencontre.

35 La structure fonctionnelle de l'activité de médiation (cf. Figure 1) révèle une grande proximité avec le format classique d'une médiation (cf. Tableau 2). Le processus canonique de la médiation - sa structure fonctionnelle théorique/officielle telle qu'enseignée - comporte six étapes :

1. la phase d'accueil où sont précisés les rôles de chacun et les différentes étapes du processus ;

2. les récits initiaux: chaque partie prend la parole de manière successive pour relater la situation posant problème ;

3. la synthèse du médiateur des propos de chacune des parties;

4. le quoi : définir l'objet concret du différend;

5. le pourquoi : identifier les raisons du différend (intérêts, besoins, valeurs...) ;

6. le comment : soumettre des propositions pour sortir du différend. 
Dans le jeu de rôle (cf. Tableau 2), la phase d'accueil bien qu'elle soit très brève, est présente (phase 1), le différend est présenté (fusion des phases 2 et 4), ses raisons (fusion des phases 2 et 5) et des tentatives de résolution du différend (phase 6). La phase de reformulation n'apparaît pas en tant que telle. Aussi, on observe au cours du processus, des récurrences de phases se rapportant à la sphère socio-émotionnelle (régulation de conflits).

Tableau 2 : Structures fonctionnelles officielles versus effective de l'activité de médiation. Table 2: Official versus effective functional structures of the mediation activity

\begin{tabular}{|l|l|}
\hline Etapes d'une médiation (théorique) & Etapes de la médiation (effective) \\
\hline 1. Phase d'accueil & 1. Phase d'accueil (très réduite) \\
\hline 2. Les récits initiaux & 2.4. Présentation du différend (quoi + récits) \\
\hline 3. La synthèse du médiateur & $\begin{array}{l}\text { 2.5. Les raisons du différend (pourquoi + } \\
\text { récits) }\end{array}$ \\
\hline 4. le Quoi (définir objet du différend) & 6. Propositions pour sortir du différend \\
\hline 5. Le Pourquoi (identifier les raisons) & \\
\hline $\begin{array}{l}\text { 6. Le Comment (propositions pour sortir du } \\
\text { différend) }\end{array}$ & \\
\hline
\end{tabular}

L'évolution des positions des parties au gré de la médiation 
Figure 2 : L'évolution des attitudes au gré de la médiation. Fig. 2 : Evolution of attitudes during the mediation process
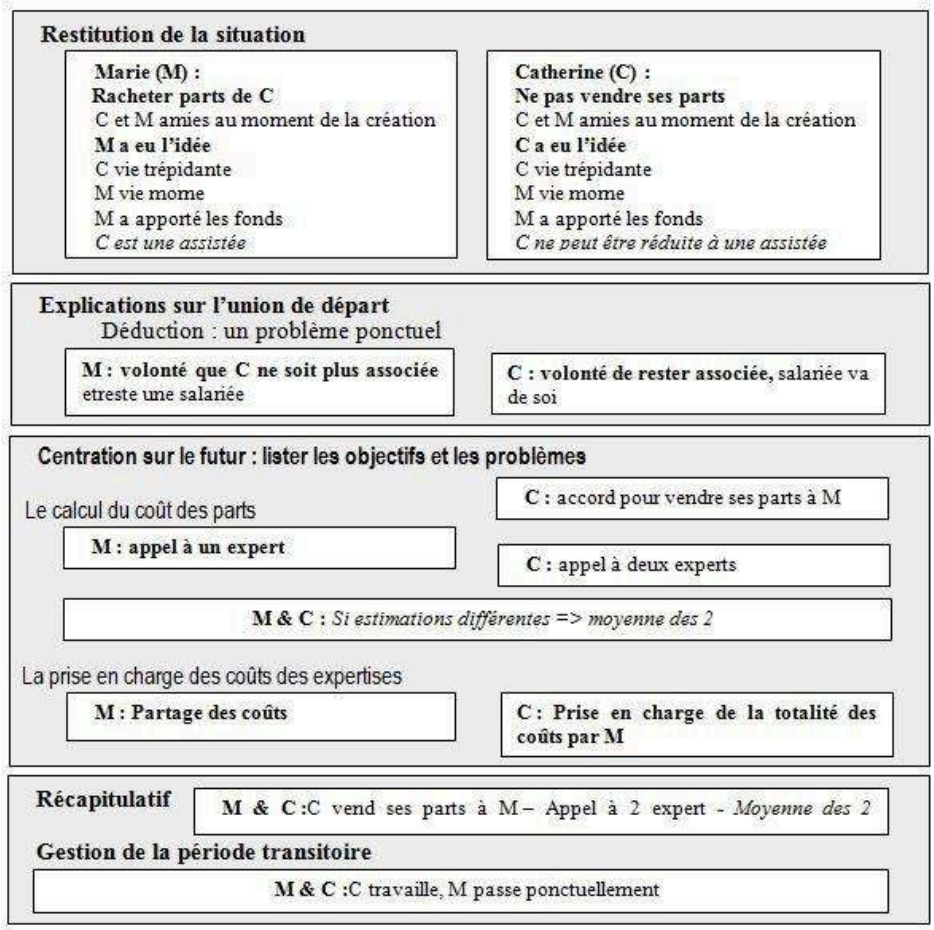

Date de la prochaine rencontre, consignes et ordre du jour (fixer le prix des parts)

Légende : Marie (M) et Catherine (C) sont les deux parties qu'un différend oppose. Les contenus qui sont objets à désaccords sont en gras ; les contenus en italique reflètent des divergences mitigées ; en standard : les contenus sont objet d'accords. Les encadrés côte à côte restituent les attitudes des parties (registre modal : accord versus désaccord) à propos de contenus propositionnels (registre référentiel : contenu représentationnel). Par exemple concernant la proposition (registre référentiel) " $\mathrm{M}$ a eu l'idée de création du magasin », M y adhère (accord/registre modal) et C n'y adhère pas (désaccord/registre modal).

Legend: $M$ and $C$ are the two parties in disagreement. The contents that are objects of disagreement are in bold; the contents in italics are the subject of minor disagreements; contents in standard typeface are objects of agreement. For each party, the boxes that are side by side describe their attitude (modal record: agreement versus disagreement) towards the propositional content (referential record: representational content). For example, concerning the proposition (reference record) "M had the idea of creating the store", $M$ agrees (agreement/modal record) and $C$ disagrees (disagreement/modal record).

On observe ainsi des changements de position au gré de la médiation. Si Catherine au départ ne souhaite pas vendre ses parts; en phase de recherche de solutions, elle exprime un changement de position (elle est d'accord pour vendre ses parts à Marie). Lorsqu'il est question d'avoir recours à un expert pour estimer le coût des parts, rapidement les deux parties conviennent d'avoir recours à deux experts, mais ne parviennent pas à trouver un accord sur la façon de procéder dans le cas où les conclusions des deux expertises seraient différentes. Quant à la prise en charge du coût de l'expertise, aucun consensus ne sera trouvé au cours de cette séance de médiation. D'autres désaccords persistent, mais n'entravent pas le processus de médiation : par exemple, le fait que Catherine estime avoir eu l'idée d'un magasin et que Marie estime que c'est elle qui en a eu l'idée. 


\section{Les rôles accomplis}

\section{Tableau 3.}

Tableau 3 : Les rôles accomplis par l'apprenante médiatrice et les parties.

Table 3: The roles played by the trainee mediator and the parties

\begin{tabular}{|c|c|c|c|}
\hline \multicolumn{2}{|c|}{ L'apprenante médiatrice } & \multicolumn{2}{|r|}{ Les parties } \\
\hline $\begin{array}{l}\text { Alloue les prises de } \\
\text { parole }\end{array}$ & & Informent & "je gérais tout $"$ \\
\hline Explicite le contrat & $\begin{array}{l}\text { "je ne suis pas là pour } \\
\text { trouver une solution } "\end{array}$ & Argumentent & $\begin{array}{l}\text { (contexte: elle serait } \\
\text { incapable de reprendre le } \\
\text { magasin) } \\
\text { « elle n'est pas compétente })\end{array}$ \\
\hline $\begin{array}{l}\text { Conduit la discussion } \\
\text { (initie les phases) }\end{array}$ & & $\begin{array}{c}\text { Contre- } \\
\text { argumentent }\end{array}$ & $\begin{array}{l}\text { (tu ne t'impliques pas) " } \mathrm{j} \text { 'ai } \\
\text { mi les fonds, je m'occupe du } \\
\text { financier } »\end{array}$ \\
\hline $\begin{array}{c}\text { Soumet sa } \\
\text { compréhension (via } \\
\text { des reformulations, } \\
\text { des synthèses) }\end{array}$ & $\begin{array}{l}\text { (alors) "au départ ça } \\
\text { allait bien " }\end{array}$ & $\begin{array}{l}\text { Décrivent des } \\
\text { états } \\
\text { émotionnels }\end{array}$ & $\begin{array}{l}\text { "c'était bien, on était } \\
\text { amies" }\end{array}$ \\
\hline $\begin{array}{l}\text { Soumet des } \\
\text { déductions }\end{array}$ & $\begin{array}{l}\text { "donc c'est un problème } \\
\text { ponctuel " }\end{array}$ & $\begin{array}{l}\text { Expriment des } \\
\text { états } \\
\text { émotionnels }\end{array}$ & $\begin{array}{l}\text { "JE NE METTRAI PAS UN } \\
\text { EURO }\end{array}$ \\
\hline Recentre & $\begin{array}{l}\text { "s'il vous plait, je } \\
\text { voudrais qu'on liste les } \\
\text { problemes" }\end{array}$ & $\begin{array}{l}\text { Attaquent, } \\
\text { insultent, } \\
\text { dénigrent }\end{array}$ & $\begin{array}{l}\text { "elle est instable " " } \text { t'es }^{\prime} \\
\text { incapable de gérer le magasin } \\
\text { seule }\end{array}$ \\
\hline $\begin{array}{l}\text { Questionne (demande } \\
\text { des explications, des } \\
\text { approfondissements...) }\end{array}$ & $\begin{array}{l}\text { "vous voulez prendre les } \\
\text { rennes, vous ne risquez pas } \\
\text { de perdre la clientele? }\end{array}$ & S'opposent & $\begin{array}{l}\text { "cंest pas moi qui ai } \\
\text { demandé une médiation } * \text { « JE } \\
\text { NE METTRAI PAS UN } \\
\text { EURO }\end{array}$ \\
\hline \multirow[t]{3}{*}{$\begin{array}{c}\text { S'oppose aux } \\
\text { dénigrements, insultes }\end{array}$} & 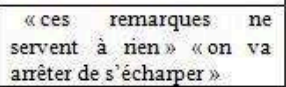 & Menacent & $\begin{array}{l}\text { "je ne vends pas mes parts, } \\
\text { je reste salariée et ta vie sera } \\
\text { un enfer» }\end{array}$ \\
\hline & & $\begin{array}{l}\text { Demandent à } \\
\text { prendre la } \\
\text { parole }\end{array}$ & $\begin{array}{l}\begin{array}{l}\text { «j'aimerais prendre la } \\
\text { parole }\end{array}\end{array}$ \\
\hline & & $\begin{array}{l}\text { Prennent la } \\
\text { parole sans la } \\
\text { demander }\end{array}$ & \\
\hline
\end{tabular}

Légende : l'emploi des majuscules traduit une forte intensité.

Legend: the use of capital letters translates strong intensity

L'étude des rôles révèle que certains ne sont pas prescrits dans le contrat de communication externe (comme insulter, dénigrer, prendre la parole de manière intempestive). Ces comportements offensifs ont contraint la médiatrice à faire des rappels à l'ordre.

Globalement les rôles accomplis par l'apprentie médiatrice correspondent à l'attendu.

\section{Les émotions}

Pour traiter de la dimension émotionnelle, nous utilisons des séquences extraites du jeu de rôle comportant des interventions potentiellement conflictuelles. Nous mettons en exergue les différents formats mobilisés pour produire des attaques comme dénigrer, insulter : l'adressage peut être direct (exemple: «t'es incapable de gérer le magasin seule ») ou indirect via des tropes communicationnels ${ }^{1}$ (exemple : « elle va de fleur en fleur » (explicite avec métaphore), «elle cherche un mari » (explicite), «moi, je ne couche pas" (implicite). Nous montrons quels sont les effets perlocutoires de ces interventions produites en situation triadique, en nous basant sur la taxonomie des émotions de Cahour et Lancry (2011, op.cit.). Par exemple lorsque M énonce : «T'es incapable de gérer le magasin seule », elle suscite une émotion primaire négative chez 
C (la honte) et une émotion complexe négative chez la médiatrice (sentiment de défiance vis-à-vis de $C$ qui peut conduire la médiatrice - en référence aux théories implicites de la personnalité (Bruner \& Tagiuri, 1954) - à affecter d'autres traits de personnalité comme : inconsistante, non fiable, imprévisible...).

La mise en évidence de tropes communicationnels a également pour objectif de montrer que le placement des parties de façon à ce qu'elles ne communiquent pas directement entre elles, n'est pas suffisante pour empêcher les attaques puisque les tropes ont pour caractéristique d'être adressés à la personne qui n'est pas la première destinataire du message.

Nous traitons également la séquence qui amorce les premiers échanges de la médiation, car l'apprenante médiatrice s'y trouve contestée. Elle demande à l'une des parties d'exposer la situation, cette dernière s'y refuse avançant que ce n'est pas elle qui a demandé la médiation. Nous étudions cette séquence sur la base des travaux de Kerbrat-Orecchioni (1988, op. cit.) et de Goffman (1974) traitant respectivement des positions hautes versus basses dans l'espace de l'interlocution et de leur incidence sur l'image du moi (la face) des protagonistes.

Apprenante médiatrice: (...) donc vous allez me dire ce qui vous amène, Mme $C$ je vous laisse prendre la parole.

Mme C : Non ce n'est pas moi qui ai demandé la médiation, c'est à $\mathrm{M}$ de prendre la

parole.

Apprenante médiatrice : D'accord, Mme M

Mme $\mathrm{M}$ : donc euh au départ (...)

L'apprenante médiatrice énonce une requête d'action adressée à C. La suite préférée d'une requête est qu'au deuxième tour, celle-ci soit satisfaite par le destinataire (Levinson, 1983). Ce n'est pas le cas ici. Mme C, à qui l'intervention est adressée, communique au deuxième tour qu'elle ne va pas satisfaire l'acte et l'exprime par un «non » sans préambule qui aurait pu atténuer l'attaque. On note néanmoins que dans la foulée elle communique la raison pour laquelle elle ne prendra pas la parole en premier. Dans son univers cognitif, la première prise de parole doit être faite par la personne qui a demandé la médiation ; comme ce n'est pas elle qui en a fait la demande, elle décline l'invitation de prise de parole. Dans les termes de la logique interlocutoire (Trognon, \& Brassac, 2012, op. cit.), l'allocutaire fait état d'une condition préparatoire non remplie (le premier à intervenir doit être celui qui a demandé la médiation). Manifestement, cette règle (celui qui demande la médiation doit débuter) n'intégrait pas l'univers cognitif de l'apprenante médiatrice. Au niveau relationnel, par cette intervention, C communique que la médiatrice n'a pas l'autorité pour imposer qui va prendre la parole - ce qui peut être problématique pour la suite, car si la médiatrice est contestée dans son rôle d'animatrice, cela peut complexifier voire altérer la conduite $\mathrm{du}$ processus de médiation. L'apprenante médiatrice subit ainsi une attaque qui potentiellement peut affecter son image du moi ; sa position est basse dans l'espace de l'interlocution. On notera qu'au troisième tour, l'apprenante médiatrice capitule (" d'accord») et applique la règle imposée par C en donnant la parole à Mme $\mathrm{M}$ - cette dernière étant à l'origine de la médiation. Cette capitulation renforce sa position basse.

\section{3. Étude des discussions collectives}

La discussion consécutive au jeu de rôle a été enregistrée, les allo-confrontations ont été filmées. Les discussions n'impliquant pas la psychologue ergonome ont fait l'objet 
de prise de notes en temps réel par cette dernière afin qu'elle puisse plus facilement se remémorer des propos à retenir pour y associer des résultats d'analyse lors de la phase d'allo-confrontation enrichie des résultats. Ce sont donc des idées émises au sens de Negura (2006) qui sont identifiées en situation et prises en note; en d'autres termes, des contenus propositionnels (dimension référentielle) couplés à des attitudes (dimension modale). Des transcriptions ont été effectuées dans l'après-coup par prélèvement - en l'occurrence ont été transcrites littéralement les idées produites par le collectif qui ont été reprises par la psychologue ergonome pour présenter ses résultats, ainsi que les interventions du collectif en réaction à la restitution de ces résultats.

Pour rendre compte de l'apport des résultats d'analyse sur les productions collectives, nous allons tout d'abord rendre compte des productions émises en discussion collective consécutive au jeu et celles produites en allo-confrontation sans la présentation des résultats, puis les productions émises en réaction à la restitution des résultats d'analyse.

\subsubsection{Les productions consécutives au jeu de rôle sans restitution de résultats}

Suite au jeu de rôle, la formatrice à la médiation affecte la parole à l'apprentie médiatrice qui a pour consigne de communiquer ses impressions, son sentiment quant à la situation qu'elle venait de vivre. Celle-ci communique qu'elle a dû se refréner : « on a envie de proposer des solutions, parce qu'on voulait que ça avance »-ce qui traduit qu'elle a bien compris que le médiateur n'est pas là pour proposer des solutions, mais pour guider les parties pour qu'elles-mêmes trouvent des solutions consensuelles. Elle a néanmoins le sentiment de ne pas avoir joué son rôle: «impression que la situation a avancé mais pas grâce au médiateur ", "je me sentais impuissante »; et qu'elle a été absorbée par la régulation de conflits au détriment de la conduite d'un processus devant mener les parties à trouver des solutions : « (...) pas le sentiment d'être dans la réflexion, on était dans l'affectif, dans le règlement de compte ».

48 La parole est ensuite donnée à Catherine qui communique qu'elle a l'impression d'une évolution. Marie prend ensuite la parole et exprime que, pour elle, c'est du perdantperdant ; elle a le sentiment que rien n'est acquis.

49 La parole est ensuite donnée aux observateurs. Les observateurs s'accordent sur le fait que trop d'attaques (insultes, dénigrements...) ont eu lieu. Ils considèrent ces attaques comme un frein au processus de médiation. Certains proposent que la disposition spatiale soit revue pour éviter que les parties soient face à face, ce qui devrait réduire les attaques. Ils notent qu'il n'y a pas eu de travail sur la ré-entente : «il faudrait des moyens pour travailler sur la ré-entente en les ramenant sur des points positifs, elles ne s'écoutent pas, elles campent sur leur position ». Pour pallier ce problème, ils font des propositions : «il faudrait fixer des règles au départ et les prévenir qu'elles sont contraintes d'écouter l'autre ».

Que ce soit suite au jeu de rôle ou en allo-confrontation, l'étude des productions des apprenants (acteurs du jeu de rôle et observateurs) révèle l'emploi de nombreux modalisateurs d'incertitude (je crois, j'ai le sentiment que, on a l'impression...). 


\subsubsection{Les discussions en allo-confrontation sans restitution de résultats}

51 L'allo-confrontation sans intervention du psychologue ergonome est animée par la formatrice à la médiation. Les apprenants abordent à nouveau la question des conflits et leur régulation sur la base de séquences visionnées.

Une apprenante mentionne que des accords qui semblaient actés, finalement ne le sont pas. Elle reprend pour illustrer ses propos, l'énoncé de Catherine : «je ne revends pas mes parts, je reste salariée et ta vie sera un enfer » et rappelle qu'à ce moment de la médiation, Catherine avait pourtant déjà manifesté qu'elle était d'accord pour vendre ses parts. Cette intervention conduira le collectif à s'interroger sur les raisons pouvant expliquer des retours en arrière et avance la possibilité que certaines phases auraient été clôturées trop rapidement.

53 La discussion se poursuit avec l'intervention d'un formé qui pose que «la médiatrice n'a pas assez travaillé sur la ré-entente des parties ", s'engage alors une discussion sur le rôle de la médiatrice.

54 Une fois que plus personne ne souhaite s'exprimer, la psychologue ergonome est invitée à restituer les résultats d'analyse du jeu de rôle.

\subsubsection{Les discussions en allo-confrontation en réaction à la restitution des résultats}

Pour cette restitution, la psychologue ergonome choisit d'articuler les résultats à des propos tenus par les apprenants lors des discussions collectives précédentes (cf. Tableau 4). L'ordre de présentation dans le tableau respecte la chronologie des propos. 
Tableau 4 : Injection des résultats d'analyse et leur impact sur la discussion. Table 4: Injection of the results of the analysis and their impact on the discussion

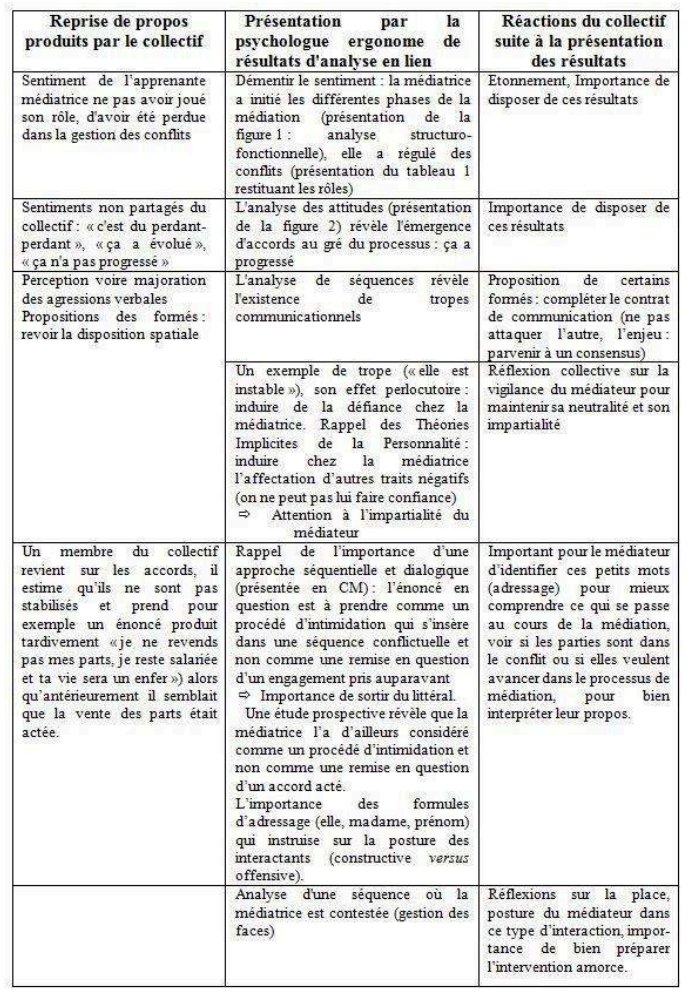

Légende : La première colonne restitue les propos produits en discussion collective retenus par la psychologue ergonome, la deuxième restitue les résultats d'analyse et la troisième, la réaction du collectif aux résultats restitués. Ainsi, chaque ligne restitue les propos collectifs repris (première case) pour chaque résultat d'analyse restitué (deuxième case) et les propos produits en réaction par les apprenants (troisième case).

Legend: The first column quotes statements produced during collective discussion and selected by the psychologist ergonomist, the second quotes the results of the analysis and the third the reaction of the group. Each line therefore quotes reiterated collective statements (first box) for each reiterated analysis result (second box) and the comments produced by the learners in response (third box).

La psychologue ergonome débute en rappelant le sentiment de la médiatrice de n'avoir pas fait avancer les choses, de n'avoir pas été dans la réflexion, mais avoir été noyée dans la régulation de conflits. Elle montre sur la base des résultats de l'analyse structuro-fonctionnelle (cf. Figure 1) que la médiatrice a été active dans la conduite du processus : elle a initié les différentes phases de la médiation au cours desquelles des modifications d'attitudes sont observées, en l'occurrence des accords sont actés (cf. Figure 2).

La psychologue ergonome propose ensuite une réflexion sur les agressions verbales qui ont été perçues voire majorées par le collectif. Elle rappelle la suggestion faite en alloconfrontation de revoir la disposition spatiale des chaises pour éviter que les parties soient face à face et que plus naturellement leur interlocuteur soit la médiatrice. L'option est pertinente et habituellement mise en œuvre; néanmoins la psychologue ergonome montre l'existence de tropes communicationnels qui ont pour caractéristique de ne pas requérir que l'interlocuteur visé par le message soit le destinataire du message explicitement adressé. À partir de l'analyse d'une séquence, elle montre que les effets perlocutoires de telles interventions affectent tant la médiatrice que l'autre partie. De cette présentation, il est ressorti collectivement que 
l'aménagement de la disposition spatiale n'était pas suffisant pour éviter les attaques portées par des tropes communicationnels. Les apprenants ont alors amorcé une réflexion sur le contrat de communication externe. Celui-ci a alors été précisé : «on n'est pas ici pour dénigrer, insulter... qui ne peut qu'altérer le processus de conciliation », «il faut mettre le focus sur les faits, sur ce que les parties souhaitent pour faire évoluer les positions vers un consensus». Un exemple de trope a été présenté. L'énoncé : « elle est instable " produit par Marie à destination de l'apprentie médiatrice est utilisé pour évoquer ses effets perlocutoires ; en l'occurrence, induire de la honte chez la destinataire indirecte et de la défiance chez l'apprenante médiatrice. Elle ajoute, en référence aux Théories Implicites de la Personnalité (Bruner, \& Tagiuri, 1954), que cela peut conduire l'apprenante-médiatrice à lui attribuer d'autres traits négatifs (instable, inconsistante...), ce qui peut affecter son impartialité. S'en suivra une discussion du collectif sur la vigilance du médiateur pour maintenir sa neutralité et son impartialité.

La psychologue ergonome reprend l'intervention de l'apprenante qui, en se basant sur l'énoncé de Catherine : «je ne revends pas mes parts, je reste salariée et ta vie sera un enfer", voulait montrer que rien n'était acquis. La psychologue ergonome fait remarquer la forte intensité de l'énoncé de Catherine - intensité non restituée par l'apprenante. La psychologue ergonome revient sur l'importance d'une approche pragmatique et séquentielle du discours (importance de se dégager du littéral), que dans le contexte d'énonciation, Catherine et Marie sont engagées dans un échange conflictuel, que l'intervention de Catherine marquée par une forte intensité est davantage à considérer comme une menace, un procédé d'intimidation plutôt qu'un retour sur ce qui a été acté antérieurement. D'ailleurs la psychologue ergonome montre qu'une approche prospective $\mathrm{du}$ discours permet d'observer que l'apprenante médiatrice a géré dans l'espace de l'interlocution cette intervention comme un procédé d'intimidation - et pas comme une remise en question d'un accord antérieur puisqu'elle réagit à cet échange en demandant aux parties de garder leur calme. Suite à ces propos, un apprenant demande comment identifier ces séquences conflictuelles. La psychologue ergonome revient sur l'importance de l'intensité de l'acte qui est un marqueur d'expression émotionnelle (Plantin, 2011), sur les formules d'adressage (elle, madame, prénom) qui peuvent donner des indications sur la proximité relationnelle (Marcoccia, 2007, op.cit.) et sur la posture des interactants en situation - posture constructive versus offensive/défensive (Cooke, \& Szumal, 1994). Un membre du collectif poursuit en confirmant l'importance d'« identifier ces petits mots pour mieux comprendre ce qui se passe au cours de la médiation, voir si les parties sont dans le conflit ou si elles veulent avancer dans le processus de médiation, pour bien interpréter leur propos ".

Enfin, la psychologue ergonome poursuit en présentant la séquence qui n'a pas interpelé le collectif lors de l'allo-confrontation. Au cours de cette séquence qui amorce les premiers échanges, l'apprentie médiatrice est contestée : elle demande que l'une des parties expose la situation, celle-ci s'y refuse avançant que ce n'est pas elle qui a demandé la médiation. Après avoir présenté l'analyse de la séquence et son impact sur l'image du moi (Goffman, 1974, op.cit.) de l'apprenante médiatrice, des réflexions collectives vont porter sur la place du médiateur dans ce type d'interaction et sur la nécessité de préparer en amont son intervention amorce. 


\section{Discussion} visant à promouvoir la réflexivité des apprenants. Effectivement, en référence aux travaux de Bouissou et Brau-Anthony (2005, op. cit.), nous avons effectivement :

- un support propice à discussion : l'activité utilisée a été produite en jeu de rôle par des novices en matière de médiation. Le jeu de rôle renvoie à une simulation de situation (Béguin, \&Weill-Fassina, 1997) qui permet une production réaliste qui émerge et existe par et au moyen de l'investissement des acteurs qui y ont pris part (Dubey, 1997). Dans le processus de formation mis en place, l'utilisation du jeu de rôle présente plusieurs avantages. Il permet à l'apprentie médiatrice de s'exercer à la pratique de la médiation - au cours de cette formation globale à la médiation, chaque apprenant aura joué au moins une fois le rôle de médiateur; d'ailleurs la certification est fournie sur la base d'une mise en situation (jeu de rôle sur la base d'un scénario imposé) évaluée par un jury. Il constitue une ressource exploitable en formation dans le sens où son format (structure fonctionnelle) est proche de l'attendu. Enfin les dysfonctionnements observés (récurrence des agressions, processus de régulation infructueux...) suscitent discussion et réflexion ;

- des savoirs formels: les savoirs académiques sur la médiation mobilisés par la formatrice pour guider, orienter les discussions, auxquels s'ajoute un cadre interprétatif permettant d'approcher sous différents angles (structuro-fonctionnel, relationnel, émotionnel) l'interaction communicationnelle. Ce cadre a fait l'objet d'un cours magistral ;

- des discussions collectives sur l'activité produites consécutivement à l'activité puis en situation d'allo-confrontation. Dans notre dispositif, en seconde partie de l'alloconfrontation, la psychologue ergonome enrichit la discussion, des résultats de l'analyse du jeu de rôle mobilisant le nouveau cadre.

61 À travers cette étude, nous observons que le recours à ce cadre interprétatif pour l'analyse des productions langagières émises en jeu de rôle a permis d'enrichir la discussion collective. En effet, la comparaison des productions en discussions exclusivement animées par la formatrice, de celles enrichies des résultats d'analyse du versant communicationnel de l'activité a permis d'identifier ce que le collectif ne perçoit pas d'emblée : en l'occurrence, l'impact de suites non préférées sur les faces (images du moi), les tropes communicationnels, le processus de médiation dans sa dimension structuro-fonctionnelle, l'importance en amont d'expliciter le contrat de communication.... Aussi, cet enrichissement a permis aux apprenants d'aller plus loin dans la réflexion, de trouver des solutions tenables pour éviter certains dysfonctionnements (revoir la disposition spatiale et surtout compléter le contrat de communication externe) et de donner une assise à certaines de leurs impressions via notamment la mise en exergue des accords qui se sont succédé au gré du processus de médiation.

Si l'analyse permet d'éclairer l'activité communicationnelle sous différents angles, elle ne permet d'accéder qu'à ce qui laisse des traces dans l'espace de l'interlocution - qui a été produit, co-construit et acté dans cet espace. Elle ne permet pas d'accéder à l'expérience vécue des protagonistes. Échappent alors à l'analyste: les émotions, intentions, désirs, croyances, stratégies... qui ont été éprouvés/mobilisés au cours de l'activité, mais qui n'ont pas laissé de traces dans le discours - ces éléments peuvent être pré-réfléchis, non conscientisables ou sciemment camouflés (Cahour, 2006, op. cit.). De fait, la psychologue ergonome lors de la restitution de certains résultats a été parfois 
conduite à utiliser des modalisateurs d'incertitude, en l'occurrence lorsqu'il a été question de traiter des émotions et des tropes communicationnels. Par exemple, concernant l'énoncé produit par une des parties : «JE NE METTRAI PAS UN EURO », cet énoncé produit avec une forte intensité traduit l'expression d'une émotion (Plantin, 2011, op. cit.) ; pris dans son contexte d'énonciation et en référence à Cahour et Lancry (2011, op. cit.), cet énoncé permet l'expression d'une émotion primaire de colère et vise "mais à vérifier (modalisateur d'incertitude)" à intimider l'autre partie (effet perlocutoire). Les questions auxquelles on ne peut répondre sur cette seule base sont de ce type : la locutrice était-elle véritablement en colère? N’a-t-elle pas simulé cette colère pour intimider l'autre partie? Éprouvait-elle d'autres affects? Et si l'on se tourne vers l'allocutaire, a-t-il identifié cette colère? A-t-il perçu uniquement cette colère ou y a-t-il vu également l'emploi d'une stratégie d'intimidation? Qu'éprouve-t-il à ce moment-là ? etc.

Concernant les tropes communicationnels, lorsque la psychologue ergonome reprend les propos de Marie adressés à l'apprentie médiatrice : « elle (Catherine) va de fleur en fleur ", elle pose en référence aux travaux de Kerbrat-Orecchioni $(1988$, op. cit.) et de Cahour et Lancry (2011, op. cit.) que cet acte, qui communique que Catherine est volage, a (" potentiellement »/modalisateur d'incertitude) pour effets perlocutoires, d'induire une émotion primaire de honte chez Catherine et une émotion complexe (un sentiment de défiance à l'égard de Catherine) chez l'apprentie médiatrice. Comme précédemment, il est impossible sur la base des seules productions langagières, de savoir si Marie avait bien ces deux intentions: induire de la honte chez le destinataire indirect et un sentiment de défiance chez le destinataire direct. Quant à l'apprentie médiatrice, a-telle identifié une stratégie de discréditation? A-t-elle éprouvé ce sentiment de défiance à l'égard de Catherine ? Quant à Catherine, a-t-elle éprouvé de la honte?

Ainsi, si l'analyse permet d'éclairer l'activité communicationnelle sous différents angles, elle présente des limites dont certaines pourraient être dépassées par l'emploi de la méthode de l'entretien d'explicitation en auto-confrontation (telle qu'utilisée par Cahour, 2006, op. cit.) auprès de chacun des acteurs du jeu de rôle. L'auto-confrontation (visualisation de la vidéo de sa propre activité) constitue un support privilégié pour accéder à l'expérience vécue des acteurs, définie comme «le flux d'actions, émotions, pensées et sensations perceptives qui émergent dans le cours de l'activité » (Cahour, Salembier, \& Zouinar, 2016, p. 260). Les relances propres à l'entretien d'explicitation permettent la restitution détaillée des expériences vécues dans leurs dimensions émotionnelle et corporelle et d'accéder à des affects pré-réfléchis (Cahour et al., 2016). Cette méthodologie "permet de saisir quelque chose de la part non observable de l'activité vécue et le sens que les sujets en produisent au fil de l'action, d'une manière inaccessible à l'observation directe du sujet et de sa conduite (...), (des éléments) qui relève(nt) d'un vécu phénoménologique qui n'est ni visible ni partagé. » (Cahour, \& Licoppe, 2010, op. cit., p. 249).

Les productions émises en entretien d'auto-confrontation permettraient ainsi d'enrichir la compréhension de l'activité de médiation en considérant le vécu subjectif des acteurs au cours du jeu de rôle et d'ouvrir sur une part cachée de l'activité (les états mentaux et émotionnels camouflés et pré-réfléchis). 


\section{Conclusion}

$\mathrm{du}$ sens commun (les praticiens savent généralement que certaines actions procèdent d'une décision réfléchie, d'autres relèvent d'automatismes, que l'action peut s'accompagner d'émotions, etc. ; Perrenoud, 2004, op. cit.). Il peut également mobiliser des connaissances propres à son expérience et à sa culture professionnelles (Perrenoud, 1999, op. cit.). Mais dès les années 99, Perrenoud (1999, op. cit.) suggère la mobilisation d'autres cadres pour interpréter les pratiques et notamment de recourir à des cadres provenant des sciences humaines et sociales. C'est dans cette perspective que nous nous situons en proposant un cadre interprétatif mettant le focus sur la dimension communicationnelle des activités professionnelles qui s'expriment à travers des interactions langagières. Ce cadre a déjà été utilisé pour enrichir la compréhension d'activités produites en entretiens d'annonce de diagnostics médicaux (Saint-Dizier de Almeida, 2013), en discussions à visée philosophique en école élémentaire (Saint-Dizier de Almeida, Specogna, Luxembourger, 2016 ; Saint-Dizier de Almeida, Colletta, AuriacSlusarczyk, Specogna, Simon, Fiema et al., 2016), lors d'une table ronde (Saint-Dizier de Almeida, Auriac-Slusarczyk, Roland-Lévy, \& Gunzburger, 2018). Il a été mobilisé pour développer le support d'une formation en ligne à la conduite d'entretien d'annonce médecin-patient (Saint-Dizier de Almeida, 2013, op.cit.) permettant l'acquisition de compétences communicationnelles (Saint-Dizier de Almeida, \& Agnoletti, 2015). Dans cette étude, il est mobilisé dans un dispositif de formation pour améliorer la compréhension de l'activité de médiation civile et commerciale et enrichir les réflexions des apprenants en discussion collective. Il a fait l'objet d'une présentation théorique, puis a été utilisé pour l'analyse de la transcription d'un jeu de rôle simulant une médiation. Les résultats d'analyse ont été restitués aux apprenants en discussion collective.

Cette étude présente de nombreux liens avec la recherche-action de Teiger et Laville (1991) qui, dans une optique formative, visait à faire acquérir aux membres de CHSCT (Comité Hygiène et Sécurité des Conditions de Travail), la pratique de l'analyse ergonomique du travail - un cadre interprétatif issu de la psychologie ergonomique. Comme ces chercheurs, nous nous sommes engagées dans une recherche-action à visée formative. Dans ce cadre, la recherche-action est un processus impliquant les participants de la scène éducative (apprenants, formateurs); elle vise généralement l'amélioration des pratiques grâce aux expériences des apprenants éclairées et nourries des savoirs théoriques en cours (Catroux, 2002). La recherche-action repose sur la collaboration entre les différents partenaires et ne peut se concevoir sans la négociation en commun du plan d'action (Catroux, 2002, op. cit.). Dans notre étude, les partenaires sont les suivants : le formateur (la formatrice à la médiation), le chercheur (la psychologue ergonome), les responsables de la formation et les apprenants. Le dispositif et le processus de formation ont été définis en concertation par la formatrice à la médiation et la psychologue ergonome. Ils ont été soumis d'une part aux responsables de la formation qui l'ont validé et ont aménagé les deux demi-journées en conséquence, et d'autre part, aux apprenants qui ont accepté le dispositif et les enregistrements à des fins de recherche. Lors de la formation, le rôle principal du formateur (la formatrice à la médiation) et du chercheur (la psychologue ergonome) 
était de favoriser la réactivité des apprenants, de faire émerger des analyses poussées (Catroux, 2002, op. cit.).

Dans cette étude, comme dans celle de Teiger et Laville (1991, op. cit.), le matériau de formation (hormis le cadre interprétatif) est constitué grâce et au moyen de la formation en déploiement. En effet, le jeu de rôle qui a été analysé par la psychologue ergonome a été produit par trois apprenants volontaires sur la base de consignes et d'un scénario élaborés par la formatrice à la médiation; les propos auxquels ont été adjoints les résultats d'analyse ont été produits par les apprenants grâce au guidage actif de la formatrice à la médiation. Articuler les résultats aux productions des apprenants était essentiel ; cette pratique relève de l'étayage (Bruner, 1983) et permet de travailler au niveau de la zone proximale des apprenants, ce qui concourt à davantage d'appropriations (Vygostki, 1997). Teiger et Laville procèdent également de cette façon : "c'est à partir des faits rapportés (par les apprenants) que confrontation, synthèse, explication peuvent se réaliser et qu'un processus de transformation des représentations peut s'enclencher chez les participants » (Teiger, \& Laville, 1991, p. 58).

Aussi, comme pour Teiger et Laville (1991, op.cit.), l'enjeu n'était pas de leur faire acquérir le cadre interprétatif et son emploi, mais tout du moins de les sensibiliser au cadre en leur montrant qu'il peut éclairer sous de nouveaux angles l'activité de médiation et donner une assise à certaines de leurs impressions. L'étude des productions collectives en réaction aux résultats permet de cerner l'appropriation de résultats restitués et la mobilisation de certains concepts propres au cadre (emploi des notions de "contrat de communication » et "d'adressage »). Néanmoins, les autres sessions de formation mobilisant les jeux de rôle n'ayant pas été enregistrées, nous ne pouvons davantage nous avancer quant à la référence à ce cadre lors des discussions consécutives à ces jeux de rôle ayant eu lieu à la suite. On peut néanmoins rapporter le sentiment de la formatrice à la médiation qui estime que le niveau des discussions de cette promotion d'apprenants était plus élevé que celui des autres promotions qu'elle avait déjà eues en charge.

\section{BIBLIOGRAPHIE}

Austin, J. (1962). How to do things with words. Oxford : Oxford University Press.

Bales, R. F. (1972). Rôles centrés sur la tâche et rôles sociaux dans des groupes ayant des problèmes à résoudre. In A. Lévy (Ed.), Psychologie sociale (pp. 263-277). Paris : Dunod.

Béguin, P., \& Weill-Fassina A. (1997). De la simulation des situations de travail à la situation de simulation. In P. Béguin \& A. Weill-Fassina (Eds.), La simulation en ergonomie : connaître, agir et interagir (pp. 5-28). Toulouse : Octarès.

Bouissou, C., \& Brau-Antony, S. (2005). Réflexivité et pratiques de formation. Regards critiques. Carrefours de l'éducation, 2(20), 113-122.

Boutinet J.-P. (1998). L'immaturité de la vie adulte. Paris : Presses Universitaires de France. DOI : 10.3917/puf.bouti.1999.01 
Brassac, C. (2001). L'interaction communicative, entre intersubjectivité et interagentivité.

Langages, 144, 39-57.

Bruner, J. (1983). Savoir faire Savoir dire. Paris : Presses Universitaires de France.

Bruner, J. (1996). L'éducation, entrée dans la culture. Paris : RETZ.

Bruner J.S., \& Tagiuri R. (1954). The Perception of people. In G. Lindzey (Ed.), Handbook of Social Psychology, vol. 2. (pp. 634-654). Cambridge, Massassuchetts : Addison-Wesley.

Butow, P., Cockbum, J., Girgis, A., Bowman, D., Schofield, P., D’Este, C., Stojanovski, E., Tassersall, \& M.H.N. and the CUES team (2008). Increasing oncologists' skills in eliciting and responding to emotional cues: evaluation of a communication skills training program. Psycho-Oncology, 17, 209-218.

Cahour, B. (2006). Les affects en situation d'interaction coopérative : proposition méthodologique. Le Travail Humain, 69(4), 379-400.

Cahour, B., \& Licoppe, C. (2010). Confrontation aux traces de son activité. Compréhension, développement et régulation de l'agir dans un monde de plus en plus réflexif. Revue d'Anthropologie des Connaissances, 4(2), 243-253.

Cahour, B., \& Lancry, A. (2011). Émotions et activités professionnelles et quotidiennes. Le Travail Humain, 74(2), 97-106.

Cahour,B., Salembier, P., \& Zouinar, M. (2016). Analyzing lived experience of activity. Le Travail Humain, 79(3), 259-284.

Camus, O. (1999). Les interactions langagières. In J.-P. Pétard (Ed.), Psychologie sociale (pp. 259-316). Rosny : Bréal.

Catroux, M. (2002). Introduction à la recherche-action : modalités d'une démarche théorique centrée sur la pratique. Cahiers de l'APLIUT, 11(3), 8-20.

Cerf, M., \& Falzon, P. (Eds.). (2005) Situations de service: Travailler dans l'interaction. Paris : Presses Universitaires de France. (Coll. Travail Humain).

Charaudeau, P. (1983). Langage et discours. Paris : Hachette.

Cooke, R. A., \& Szumal, J. L. (1994). The impact of group interaction styles on problem-solving effectiveness. The Journal of Applied Behavioral Science, 30(4), 415-437.

Clot, Y. (1999). La fonction psychologique du travail. Paris : Presses Universitaires de France.

Clot, Y., Faïta D., Fernandez, G., \& Scheller, L. (2001). Les entretiens en auto-confrontation croisée : une méthode en clinique de l'activité. Éducation Permanente, 146, 17-27.

Correa Molina, E., Collin, S., Chaubet, P., \& Gervais, C. (2010). Concept de réflexion : un regard critique. Éducation et francophonie, 38(2), 135-154.

Cuenot, S., Cochand, P., Lanares, J., Feihl, F., Bonvin, R., Guex, P., \& Waeber, B. (2005). L'apport du patient simulé dans l'apprentissage de la relation médecin-malade. Pédagogie médicale, 6(4), 216-224.

Deloffre, G., \& Cachard, O., (2016). Former à la médiation civile et commerciale. Premier bilan d'une expérience certifiante de formation continue. Proceedings 6th International Biennal on negociation, Paris (p. 11). https://www.novancia.fr/documents/biennale-negociation/ Programme_International-Biennale-Negotiation-2016.pdf 
Donnay, J., \& Charlier, E. (2006). Apprendre par l'analyse des pratiques : initiation au compagnonnage réflexif. Namur : Presses Universitaires de Namur.

Dubey, G. (1997). Faire « comme si » n'est pas faire. In P. Béguin \& A. Weill-Fassina (Eds.), La simulation en ergonomie : connaître, agir et interagir (pp. 39-53). Toulouse : Octarès.

Falzon, P. (1994). Dialogues fonctionnels et activité collective. Le Travail Humain, 57(4), 299-312.

Goffman, E. (1974). Les rites d'interaction. Paris : Éditions de minuit.

Guillaumin, C. (2009). La réflexivité comme compétence : enjeu des nouvelles ingénieries de la formation. Cahiers de sociolinguistique, 14(1), 85-101.

Hoc, J.-M. (1996). Supervision et contrôle de processus. Grenoble : Presses Universitaires de Grenoble.

Kerbrat-Orecchioni, C. (1986). L'implicite. Paris : Armand Colin.

Kerbrat-Orecchioni, C. (1988). La notion de « place » interactionnelle ou les taxèmes, qu'est-ce que c'est que ça ? In J. Cosnier, N. Gelas \& C. Kerbrat-Orecchioni (Eds.), Échanges sur la conversation (pp. 185-198). Paris : Éditions du CNRS.

Kostulski, K., \& Trognon, A. (1998). Le domaine cognitif de l'interlocution : un exercice d'analyse interlocutoire d'une transmission orale dans une équipe paramédicale. In Kostulski, K. \& Trognon, A. (Eds.), Communications interactives dans les groupes de travail (pp. 59-101). Nancy : Presses Universitaires de Nancy.

Lacoste, M. (1983). Des situations de parole aux activités interprétatives. Psychologie Française, 28(3/4), 231-238.

Lancry-Hoestlandt A., \& Laville, A. (2004). Le travail. In E. Brangier, A. Lancry \& C. Louche (Eds.), Les dimensions humaines du travail (pp. 43-63). Nancy : Presses Universitaires de Nancy.

Laurent, Y., \& Saujat, F. (2015). L'intervention en milieu de travail éducatif entre visée de transformation et visée de connaissance, Carrefours de l'éducation, 39(1), 19-35.

Levinson, S. (1983). Pragmatics. Cambridge : Cambridge University Press.

Marcoccia, M. (2007). Communication électronique et rapport de places : analyse comparative de la formulation d'une requête administrative par courrier électronique et par courrier papier. SEMEN, 20. URL : http://semen.revues.org/document1075.html.

Mollo, V., \& Falzon, P. (2004). Auto- and allo-confrontation as tools for reflective activities. Applied Ergonomics, 35(6), 531-540.

Mollo, V., \& Nascimento, A. (2013). Pratiques réflexives et développement des individus, des collectifs et des organisations. In P. Falzon (Ed.), Ergonomie constructive (pp. 207-222). Paris : Presses Universitaires de France.

Negura, L. (2006). L'analyse de contenu dans l'étude des représentations sociales. SociologieS. Revue en ligne : https://sociologies.revues.org/993\#quotation

Perrenoud, P. (1999). Former les enseignants dans des contextes sociaux mouvants : pratique réflexive et implication critique. Congrès de l'APNED (Association nationale pour la recherche en éducation), Caxambu (Brasil). http://www.unige.ch/fapse/SSE/teachers/perrenoud/php_main/ php_1999/1999_26.html.

Perrenoud, P. (2001). Articulation théorie-pratique et formation de praticiens réflexifs en alternance. Alternance et complexité en formation. In P. Lhez, D. Millet, \& B. Séguier (Eds.), Alternance et complexité en formation. Éducation, Santé, Travail social (pp. 10-27). Paris : Seli Arslan. 
Perrenoud, P. (2004). Adosser la pratique réflexive aux sciences sociales, condition de la professionnalisation. Éducation permanente, 160, 35-60.

Plantin, C. (2011). Les bonnes raisons des émotions. Principes et méthode pour l'étude du discours émotionné. Bern : Peter Lang.

Prost, M., Cahour, B., \& Détienne, F. (2014). Le partage d'émotions et de connaissances sur la pratique : dynamique des échanges dans les communautés de pratiques virtuelles de professionnels. Le Travail Humain, 77(2), 177-202.

Rogers, C. (2008) [1942]. La relation d'aide et la psychothérapie. Paris : ESF. [Counseling and psychotherapy. Boston : Houghton Mifflin Co.]

Roulet, E., Auchlin, A., Moeschler, J., Rubbattel, C., \& Schelling, M. (1985). L'articulation du discours en français contemporain. Berne : Peter Lang.

Roy, A. (2008). L'oral au service des habiletés réflexives. Québec français, 149, 87-88.

Saint-Dizier de Almeida, V. (2009). La dimension relationnelle des communications : étude d'interactions homme-machine tutorielles a-didactiques. Activités, 6(2), 75-99. https:// journals.openedition.org/activites/2234

Saint-Dizier de Almeida, V. (2013). Comment améliorer la compréhension de l'entretien d'annonce de diagnostics médicaux sérieux. Activités, 10(2), 54-81. https:// journals.openedition.org/activites/740

Saint-Dizier de Almeida, V. (2015) L'utilisation conjointe de l'observation et l'auto-confrontation à travers l'étude d'une vente à domicile. Le Travail Humain, 78(4), 307-334.

Saint-Dizier de Almeida, V., \& Agnoletti, M. F. (2015). Impact of online training on delivering a difficult medical diagnosis: Acquiring communication skills. Applied ergonomics, 50, 242-250.

Saint-Dizier de Almeida, V., Auriac-Slusarczyk, E., Roland-Lévy, C., \& Gunzburger, Y. (2018). Étude d'une table-ronde traitant de l'impact de l'exploitation du gaz de charbon sur l'environnement. Cahiers Internationaux de Psychologie Sociale, 117-118(1), 47-69.

Saint-Dizier de Almeida, V., Colletta, J.-M., Auriac-Slusarczyk, E., Specogna, A., Simon, J.P., Fiema, \& G. Luxembourger, C. (2016). Study activities that take place in speech interactions: A theoretical and methodological framework. International Journal of Qualitative Studies in Éducation, 29(5), 686-713.

Saint-Dizier de Almeida, V., Specogna, A., \& Luxembourger, C. (2016). L'activité communicationnelle enseignante lors des discussions à visée philosophique. Revue Recherches en Éducation, 24, 54-64.

Schön, D. A. (1987). Educating the reflective practitioner: Toward a new design for teaching and learning in the professions. San Francisco : Jossey-Bass.

Shannon, C.E., \& Weaver, W. (1949). The Mathematical Theory of Communication. Urbana, IL : University of Illinois Press.

Six-Touchard, B. (1998). Développement de la compétence tutorale par l'auto-analyse du travail. Éducation permanente, 135, 87-98.

Smets-Gary, C., \& Becker, M. (2016). Médiation et techniques de négociation intégrative : approche pratique en matière civile, commerciale et sociale. Bruxelles : Larcier.

Teiger, C., \& Laville, A. (1991). L'apprentissage de l'analyse ergonomique du travail, outil d'une formation pour l'action. Travail et emploi, 41(1), 53-62. 
Theureau, J. (1992). Le cours d'action : analyse sémiologique : essai d'une anthropologie cognitive située. Berne : Peter Lang.

Theureau, J. (2004). Le cours d'action : méthode élémentaire. Toulouse : Octarès.

Trognon, A., \& Brassac, C. (1992). L'enchaînement conversationnel. Cahiers de Linguistique Française, 13, 76-107.

Vacher, Y. (2011). La pratique réflexive : un concept et des mises en œuvre à définir. Varia, 66, 65-78. URL : http://journals.openedition.org/rechercheformation/1133 ; DOI : 10.4000/ rechercheformation.1133

Vermersch, P. (1994, 2014). L'entretien d'explicitation. Paris : ESF.

Vinatier, I, \& Marfizi, I. (2018). L'irruption des émotions entre conseillers pédagogiques et enseignants débutants : quelle conception de formation pour les mettre à distance? In I. Vinatier, L. Filliettaz et M. Laforest (Eds.), L'analyse des interactions dans le travail ; Outil de formation professionnelle et de recherche (pp. 209-234). Dijon : Raison et passions.

Vincent, D., \& Bernard Barbeau, G. (2012). Insulte, disqualification, persuasion et tropes communicationnels : à qui l'insulte profite-t-elle ? Argumentation et Analyse du Discours. Revue en ligne : http://journals.openedition.org/aad/1252, DOI : 10.4000/aad.1252

Vion, R. (1992). La communication verbale. Paris : Hachette Supérieur.

Vygotski, L. (1997). Pensée et Langage. Paris : La Dispute.

Whalen, M. R., Zimmerman, \& D.H., Wahlen, J. (1992). Une conversation fatale. Réseaux, 10(55), 145-178.

Watzlawick, P., Beavin, J.H., \& Jackson, D.D.A. (1972). Une logique de la communication. Paris : Le Seuil.

\section{NOTES}

1. On parle de trope communicationnel lorsqu'il y a un décalage entre le destinataire apparent de l'énoncé et le destinataire réel (Kerbrat-Orecchioni, 1986 ; Vincent, \& Bernard Barbeau, 2012.)

\section{RÉSUMÉS}

L'interrogation des pratiques professionnelles par les professionnels eux-mêmes en discussion collective leur permet d'améliorer leur compréhension des activités de travail et de développer des compétences à la réflexivité. Dans le champ de l'éducation, un enjeu est de proposer de nouveaux cadres interprétatifs afin que les professionnels perçoivent leur activité sous de nouveaux angles et que les échanges sur les pratiques soient enrichis. Le but de cet article est de proposer un nouveau cadre interprétatif mobilisable pour l'étude des activités professionnelles qui se matérialisent à travers des interactions langagières. Le cadre exploite des modèles, théories, notions, méthodologies issues des sciences du langage et de la psychologie de la communication. L'enjeu de l'article est d'étudier l'apport de ce cadre dans un processus de 
formation à la médiation civile et commerciale destinée à des avocats et des notaires. Le dispositif de formation exploite la technique du jeu de rôle, les résultats d'analyse des productions émises en jeu de rôle-analyse basée sur le cadre -, une discussion collective consécutive au jeu de rôle et une discussion en allo-confrontation au cours de laquelle les résultats d'analyse mobilisant le cadre sont présentés. De cette étude, il ressort que la restitution des résultats d'analyse $\mathrm{du}$ jeu de rôle basée sur ce cadre permet d'éclairer l'activité communicationnelle sous des angles auxquels les formés n'ont pas accès d'emblée, de mettre au jour des dysfonctionnements/phénomènes restés jusque-là inaperçus. Surtout, cette restitution fournit les outils conceptuels à une réflexivité utile, le cas échéant, à l'optimisation des pratiques professionnelles.

Reflection on professional practices by professionals themselves in collective discussions allows them to improve their understanding of work activities, and to develop reflexivity skills. In the educational field, one challenge is to offer new interpretive frameworks allowing professionals to perceive their activity in a new light and enhance reflection on practices in collective discussion. The goal of this paper is to present a new interpretive framework that can be used to study professional practices that take place through linguistic interactions. This framework includes models, theories, concepts and methodologies taken from language sciences and the psychology of communication. The issue in this paper is to study how this framework contributes to the process of training lawyers and notaries in civil and commercial mediation. The training system uses: (i) the role-play technique, (ii) the results of the analysis of verbal productions voiced during role-play (analysis based on the framework), (iii) a collective discussion consecutive to the role-play and (iv) a discussion in allo-confrontation, during which the results of the analysis are presented. From this study, we can see that the restitution of the results of the role play analysis based on the framework clarifies the communicational activity from viewpoints that are not immediately accessible to the trainees. It also makes it possible to revise dysfunctions/ phenomena that have so far gone unnoticed. Above all, it provides conceptual tools for a reflexivity that might be useful in the optimization of professional practice.

\section{INDEX}

Keywords : person-to-person communication, role-play, training, reflexivity

Mots-clés : communication interpersonnelle, jeu de rôle, formation, réflexivité

\section{AUTEURS}

\section{VALÉRIE SAINT-DIZIER DE ALMEIDA}

23bd Albert $1^{\text {er }}$ BP 13397, 54015 Nancy Cedex, 2LPN, Université de Lorraine, valerie.saintdizier@univ-lorraine.fr

\section{FLORA ILARDO}

23bd Albert $1^{\text {er }}$ BP 13397, 54015 Nancy Cedex, 2LPN, Université de Lorraine, flora.ilardo@laposte.net

\section{ISABELLE SERRE}

Bout à Bout Médiation - 6 Quai Jules Ferry, 88000 EPINAL, mediationepinal@gmail.com 


\section{OLIVIER CACHARD}

13 place Carnot, CO 70026, 54035 Nancy Cedex, BETA-REGLE, Université de Lorraine olivier.cachard@univ-lorraine.fr

GUY DELOFFRE

13, rue M. Ney, 54000 Nancy, CEREFIGE - ICN Business School, guy.deloffre@icn-artem.com 